\title{
Queuing Network Models for Delay Analysis of Multihop Wireless Ad Hoc Networks *
}

\author{
Nabhendra Bisnik \\ Rensselaer Polytechnic Institute, $1108^{\text {th }}$ Street, Troy, NY, USA 12180. \\ Alhussein A. Abouzeid ${ }^{1}$ \\ Rensselaer Polytechnic Institute, $1108^{\text {th }}$ Street, Troy, NY, USA 12180.
}

\begin{abstract}
In this paper we analyze the average end-to-end delay and maximum achievable per-node throughput in random access multihop wireless ad hoc networks with stationary nodes. We present an analytical model that takes into account the number of nodes, the random packet arrival process, the extent of locality of traffic, and the back off and collision avoidance mechanisms of random access MAC. We model random access multihop wireless networks as open G/G/1 queuing networks and use the diffusion approximation in order to evaluate closed form expressions for the average end-to-end delay. The mean service time of nodes is evaluated and used to obtain the maximum achievable per-node throughput. The analytical results obtained here from the queuing network analysis are discussed with regard to similarities and differences from the well established information-theoretic results on throughput and delay scaling laws in ad hoc networks. We also investigate the extent of deviation of delay and throughput in a real world network from the analytical results presented in this paper. We conduct extensive simulations in order to verify the analytical results and also compare them against NS-2 simulations.
\end{abstract}

Key words: Ad Hoc Networks; Queuing Networks; Throughput Delay Analysis.

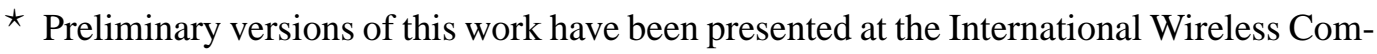
munications and Mobile Computing Conference (IWCMC), July 3-6, 2006, Vancouver, Canada and International Workshop on Wireless Ad Hoc and Sensor Networks (IWWAN), June 28-30, 2006, New York, USA (invited).

Email addresses: bisnin@rpi .edu (Nabhendra Bisnik), abouzeid@ecse.rpi.edu (Alhussein A. Abouzeid).

1 Corresponding author.
} 


\section{Queuing Network Models for Delay Analysis of Multihop Wireless Ad Hoc Networks}

\section{INTRODUCTION}

A multihop wireless ad hoc network is a collection of nodes that communicate with each other without any established infrastructure or centralized control. The transmission power of a node is limited, thus the packets may have to be forwarded by several intermediate nodes before they reach their destinations. Hence each node may be a source, destination and relay. The wireless medium is shared and scarce, therefore ad hoc networks require an efficient MAC protocol [2]. Since ad hoc networks lack infrastructure and centralized control, MAC protocols for ad hoc networks should be distributed, such as random access MAC protocols, e.g. MACA [11] and MACAW [2]. The delay and throughput of wireless ad hoc networks depend on the number of nodes, the transmission range of the nodes, the network traffic pattern and the behavior of the MAC protocol.

In this paper we investigate how the end-to-end delay and maximum achievable throughput in a random access based MAC multihop wireless network with stationary nodes depend on the number of nodes, transmission range and traffic pattern. We propose a queuing network model for delay analysis of random access multihop wireless ad hoc networks. The queuing network model proposed in this paper is unique in that it allows us to derive closed form expressions for the average endto-end delay and maximum achievable throughput. The packet delay is defined as the time taken by a packet to reach its destination node after it is generated. The average end-to-end delay is the expectation of the packet delay over all packets and all possible network topologies. Our analysis takes into account the queuing delays at source and intermediate nodes. The packets are assumed to have a fixed size and random arrival process. Moreover we also characterize how the average end-to-end delay and maximum achievable throughput vary with the degree of locality of traffic. The primary purpose of this study is not to accurately model the performance of standard protocols like IEEE 802.11 MAC (even though the results do provide a good match with $n s-2$ simulations) but to gain insights into the queuing delays and maximum achievable throughput in random access multihop wireless ad hoc networks.

Several studies have focused on finding the maximum achievable throughput and characterizing capacity-delay tradeoffs in wireless ad hoc networks e.g. $[8,10,14$, 
15]. In [10] it is shown that for a wireless network with $n$ stationary nodes, the per-node capacity scales ${ }^{2}$ as $\Theta(W / \sqrt{n \log n})$. In [14], the authors use simulations in order to study the dependence of per-node capacity on IEEE 802.11 MAC interactions and traffic pattern for various topologies like single cell, chain, uniform lattice and random network. An estimate of the expressions for one-hop capacity and upper bound of per-node throughput is obtained using the simulation results.

In [8], the authors characterize the delay-throughput tradeoffs in wireless networks with stationary and mobile nodes. It is shown that for a network with stationary nodes, the average delay and throughput are related by $D(n)=\Theta(n T(n))$, where $D(n)$ and $T(n)$ are the average end-to-end delay and throughput respectively. However the delay is defined as the time taken by a packet to reach the destination after it has left the source. Also, according to the network model, the packet size scales with the throughput. Under these assumptions the delay is simply proportional to the average number of hops between a source-destination pair. i.e. In their model, there is no delay due to queuing. If, more realistically, the packet size is assumed to be constant and the delay is defined as time taken by a packet to reach the destination after its arrival at the source, there would be queuing delays at the source and intermediate nodes.

Several recent studies have proposed queuing models for performance evaluation of the IEEE 802.11 MAC. A finite queuing model is proposed and used in [24] for evaluating the packet blocking probability and MAC queuing delays in a Basic Service Set. A queuing model for performance evaluation of IEEE 802.11 MAC based WLAN in the presence of HTTP traffic is proposed in [17]. In [18] the service time of a node, in IEEE 802.11 MAC based wireless ad hoc network, is modeled as a Markov modulated general arrival process. The resulting M/MMGI/1/K queuing model is used for delay analysis over a single hop in the network. An analytical model for evaluating closed form expression for the average queuing delay over $a$ single hop in IEEE 802.11 based wireless networks is presented in [23]. In [19], the authors use queuing theoretic approach in order to calculate the mean packet delay, maximum throughput and collision probability for an elementary four node network with hidden nodes and extend the results to linear wireless networks. It is worth noting that none of the prior works $[17-19,23,24]$ extends to a general two dimensional wireless network.

2 The asymptotic notations used in this paper have the following meanings:

- $f(n)=\Theta(g(n)) \Rightarrow \exists c_{1}, c_{2}, n_{o}>0$ s.t. $c_{1} g(n) \leq f(n) \leq c_{2} g(n) \forall n \geq n_{o}$.

- $f(n)=O\left(g(n) \Rightarrow \exists c, n_{o}>0\right.$ s.t. $0 \leq f(n) \leq c g(n) \forall n \geq n_{o}$.

- $f(n)=o\left(g(n) \Rightarrow \exists c, n_{o}>0\right.$ s.t. $0 \leq f(n)<c g(n) \forall n \geq n_{o}$.

- $f(n)=\omega(g(n)) \Rightarrow \exists c, n_{o}>0$ s.t. $0 \leq c g(n)<f(n) \forall n \geq n_{o}$. 
We propose a detailed analytic model for multihop wireless ad hoc networks based on open $\mathrm{G} / \mathrm{G} / 1$ queuing networks. We first evaluate the mean and second moment of service time over a single hop by taking into account the back off and collision avoidance mechanisms of IEEE 802.11 MAC. We then use the diffusion approximation for solving open queuing networks in order to derive closed form expression for the average end-to-end packet delay. Using the average service time of the nodes we obtain an expression for the maximum achievable throughput. We present detailed discussions on how the maximum achievable throughput obtained from our model compares with the per-node capacity of Gupta-Kumar's model. The main results of this paper are:

(1) The average end-to-end packet delay for our model is $D(n)=\frac{\rho}{\lambda \cdot(1-\hat{\rho})}$, where $\rho$ is the utilization factor of a node, $\lambda$ is the packet arrival rate at the nodes and $\hat{\rho}$ is a variable whose value depends on first and second moments of inter arrival and service times.

(2) The maximum achievable throughput in a multihop wireless ad hoc network is $\lambda_{\max }=o\left(\frac{1}{\bar{s} n r(n)^{2}}\right)$, where $\bar{s}$ is the expected number of hops between a source destination pair and $r(n)$ is the transmission radius of the nodes.

(3) When the parameters of our network model are comparable to the GuptaKumar's model [10], $\lambda_{\max }=o\left(\frac{W}{\sqrt{n \log n}}\right)$.

The analytical results are verified against extensive simulations and numerical computations. We also perform NS-2 simulations and discuss how the analytical results compare with the delay results obtained for some of the established wireless protocols.

The rest of the paper is organized as follows. In Section 2 we briefly describe the well known diffusion approximation for solving open G/G/1 queuing networks. Detailed description of the network model in Section 3 Delay and throughput analysis of multihop wireless networks is presented in Section 4. In Section 5 we present discuss intuitive interpretations of the analytical results and investigate how the results deviate from delay and throughput in a more realistic network. The comparison of the analytical and simulation results is presented in Section 6. Finally we present concluding remarks in Section 7.

\section{DIFFUSION APPROXIMATION METHOD}

The diffusion approximation [4] can be used for solving an open G/G/1 queuing network provided that all the nodes in the network are single server with first-come first-serve (FCFS) service strategy. The advantage of using the diffusion approximation in this work is that it allows us to obtain closed form expressions for the average end-to-end delay. 
In this section we briefly describe how the diffusion approximation is used to solve an open G/G/1 queuing network. (Please see [4] for details). Consider an open queuing network with $n$ service stations, numbered from 1 to $n$. The external arrival of a job is a renewal process with an average inter-arrival time of $1 / \lambda_{e}$ and the coefficient of variance of inter-arrival time equals $c_{A}$. The mean and coefficient of variance of the service time at a station $i$ are denoted by $1 / \mu_{i}$ and $c_{B i}$, respectively.

The visit ratio of a station in a queuing network is defined as the average number of times a job is forwarded by (i.e. visits) the station. The visit ratio of station $i$, denoted by $e_{i}$, is given by

$$
e_{i}=p_{0 i}(n)+\sum_{j=1}^{j=n} p_{j i}(n) \cdot e_{j}
$$

where $p_{0 i}$ denotes the probability that a job enters the queuing network from station $i$ and $p_{j i}$ denotes the the probability that a job is routed to station $i$ after completing its service at station $j$.

There are two sources of job arrivals at a station: the jobs that are generated at the station and the jobs that are forwarded to the station by other stations. The resulting arrival rate is termed the effective arrival rate at a station. The effective arrival rate at the station $i$, denoted by $\lambda_{i}$ is given by

$$
\lambda_{i}=\lambda_{e} e_{i}
$$

The utilization factor of station $i$, denoted by $\rho_{i}$, is given by

$$
\rho_{i}=\lambda_{i} / \mu_{i}
$$

The squared coefficient of variance of the inter-arrival time at a station $i$, denoted by $c_{A i}^{2}$, is approximated using

$$
c_{A i}^{2}=1+\sum_{j=0}^{n}\left(c_{B j}^{2}-1\right) \cdot p_{j i}^{2} \cdot e_{j} \cdot e_{i}^{-1}
$$

where $c_{B 0}^{2}=c_{A}^{2}$.

According to the diffusion approximation, the approximate expression for the probability that the number of jobs at station $i$ equals $k$, denoted by $\hat{\pi}_{i}(k)$, is

$$
\hat{\pi}_{i}(k)= \begin{cases}1-\rho_{i} & k=0 \\ \rho_{i}\left(1-\hat{\rho}_{i}\right) \hat{\rho}_{i}^{k-1} & k>0\end{cases}
$$

where

$$
\hat{\rho}_{i}=\exp \left(-\frac{2\left(1-\rho_{i}\right)}{c_{A i}^{2} \cdot \rho_{i}+c_{B i}^{2}}\right)
$$




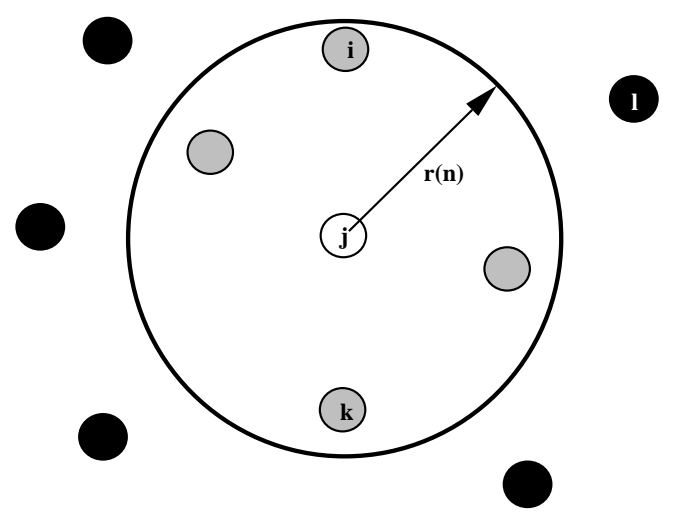

Fig. 1. A portion of a multihop wireless ad hoc network.

The mean number of jobs at a station $i$, denoted by $\overline{K_{i}}$, is

$$
\overline{K_{i}}=\rho_{i} /\left(1-\hat{\rho}_{i}\right)
$$

\section{QUEUING NETWORK MODEL}

In this section we present the network model and develop a queuing network model for multihop wireless networks. We also derive expressions for the parameters of the queuing network model.

\subsection{The Network Model}

The network consists of $n+1$ nodes, numbered 1 to $n+1$, that are distributed uniformly and independently over a torus of unit area. We assume a torus area in order to avoid complications in the analysis caused by the edge effects. Each node is assumed to have an equal transmission range, denoted by $r(n)$. Let $r_{i j}$ denote the distance between nodes $i$ and $j$. Nodes $i$ and $j$ are said to be neighbors if they can directly communicate with each other, i.e. if $r_{i j} \leq r(n)$. Let $N(i)$ denote the set of nodes that are neighbors of node $i$. All the neighbors of a node lie on a disc of area $A(n)=\pi r(n)^{2}$ centered at the node. The area $A(n)$ is termed the "communication area" of a node. The communication area is chosen such that the network is connected which ensures that $N(i) \neq \phi \forall i$. The transmission rate of each node equals $W$ bits/second.

We use a special case of the Protocol Model of interference described in [10]. If node $i$ transmits to node $j$ then the transmission will be successful only if (i) $r_{i j} \leq$ $r(n)$ and (ii) $r_{k j}>r(n)$ for every other node $k \neq i, j$ that transmits simultaneously with node $i$. In other words, node $i$ can successfully transmit a packet to node $j$ only if $i$ is a neighbor of $j$ and no other neighbor of $j$ is transmitting concurrently 
with $i$. (This is equivalent to setting $\Delta=0$ in the Protocol Model in [10]).

The traffic model for the network may be described as follows. Each node in the network could be a source, destination and/or relay of packets. Each node generates packets with rate $\lambda$ packets/sec. The delay analysis is possible for any packet generation process as long as the mean and SCV of packet inter arrival time is known. For the sake of simplicity, we assume in our model that the packet generation process at each node is an i.i.d. Poisson process. The size of each packet is constant and equals $L$ bits. When a node receives a packet from any of its neighbors, it either forwards the packet to its neighbors with probability $(1-p(n))$ or absorbs the packet with probability $p(n)$. The probability $p(n)$ is referred to as "absorption probability". In other words, the absorption probability is the probability that a node is the destination of a packet given that the node has received the packet from its neighbors. When a node forwards a packet, each of its neighbors is equally likely to receive the packet. The advantage of such a model is that we can control the locality of the traffic by varying the parameter $p(n)$. The traffic is highly localized if $p(n)$ is large while a small value of $p(n)$ implies unlocalized traffic. This would help us to characterize the effect of the locality of the traffic on the average delay and maximum achievable throughput.

For example, suppose that node $j$ in Figure 1 receives a packet from $i$. The probability that node $j$ is the destination of the packet is $p(n)$. The probability that node $j$ forwards the packet to one of its neighbors is $(1-p(n))$. Suppose node $j$ forwards the packet, then the probability that the packet is forwarded to node $k$ is $\frac{1}{|N(j)|}=\frac{1}{4}$.

We assume that each node in the network has infinite buffers which means that no packets are dropped in the network. The packets are served by the nodes on first come first serve basis.

Multihop wireless ad hoc networks can be modeled as a queuing network as shown in Figure 2(a). The stations of the queuing network correspond to the nodes of the wireless network. The forwarding probabilities in the queuing network, denoted by $p_{i j}$, correspond to the probability that a packet that is transmitted by node $i$ enters the node $j$ 's queue. Figure 2(b) shows a representation of a node in the ad hoc network as a station in the queuing network.

The end-to-end delay in a wireless network equals the sum of queuing and transmission delays at source and intermediate nodes. We will use the queuing network model shown in Figures 2(a) and 2(b) in order to mathematically analyze the endto-end delay. 


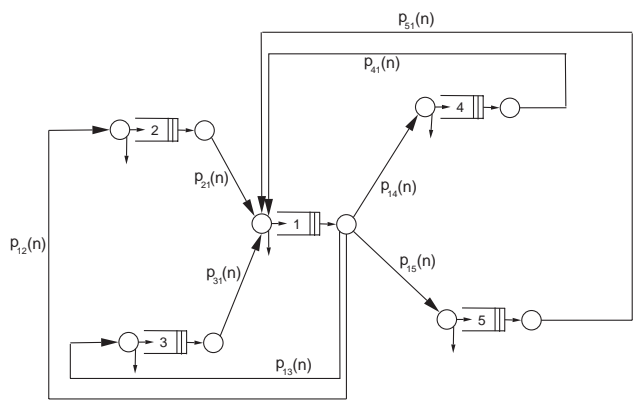

(a) Representation of multihop wireless ad hoc network as a queuing network.

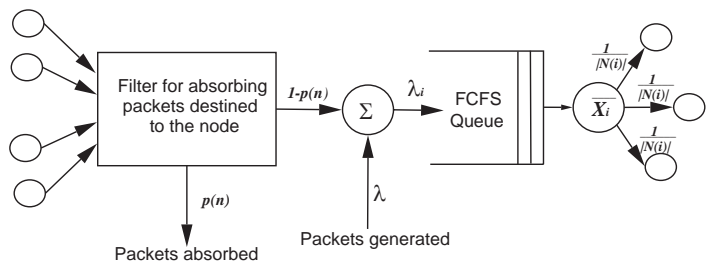

(b) Representation of a node of multihop wireless ad hoc network as a station in the queuing network.

Fig. 2. Queuing network model for multihop wireless ad hoc network.

\subsection{Parameters of the Queuing Network Model}

In this section we derive expressions for the parameters of the queuing network model of multihop wireless networks.

Lemma 1 The expected probability that a packet is forwarded from node $i$ to node $j$, denoted by $\overline{p_{i j}}(n)$, is

$$
\overline{p_{i j}}(n)= \begin{cases}\frac{1-p(n)}{n}\left(1-(1-A(n))^{n}\right) & i \neq j \\ 0 & i=j\end{cases}
$$

Proof Let $P[i \rightarrow j]$ denote the probability that a packet forwarded by node $i$ enters the queue at node $j$. We define $\beta_{i j}^{j, k}=P[i \rightarrow j|j \in N(i)| N,(i) \mid=k]$, 
$\beta_{i j}^{j}=P[i \rightarrow j \mid j \in N(i)]$ and $\alpha_{i}^{j, k}=P[|N(i)|=k \mid j \in N(i)]$. Thus

$$
\beta_{i j}^{j, k}=\frac{1}{k}(1-P[j \text { absorbs the packet }])=\frac{1-p(n)}{k}
$$

Since the nodes are uniformly and independently distributed over a unit area, the probability that a node is in neighborhood of the node $i$ equals $A(n)$. Hence $P[j \in$ $N(i)]=A(n)$ and

$$
\alpha_{i}^{j, k}=\left(\begin{array}{c}
n-1 \\
k-1
\end{array}\right)(1-A(n))^{n-k} A(n)^{k-1}
$$

Therefore,

$$
\overline{\beta_{i j}^{j}}=E\left[\beta_{i j}^{j}\right]=\sum_{k=1}^{n} \beta_{i j}^{j, k} \alpha_{i}^{j, k}=\frac{1-p(n)}{n A(n)}\left(1-(1-A(n))^{n}\right)
$$

Also according to the model node $i$ cannot forward a packet to node $j$ unless $j \in$ $N(i)$. Hence $E[P[i \rightarrow j] \mid j \notin N(i)]=0$. So the expected forwarding probability is given by

$$
\overline{p_{i j}}(n)=\overline{\beta_{i j}^{j}} P[j \in N(i)]=\frac{1-p(n)}{n}\left(1-(1-A(n))^{n}\right)
$$

Lemma 2 The expected visit ratio of node $i$, denoted by $\overline{e_{i}}$, is given by

$$
\overline{e_{i}}=\frac{1}{(n+1) p(n)} \forall i
$$

Proof The visit ratio of a node in the queuing network is given by (1). Taking expectation of both sides of the equation we have,

$$
\overline{e_{i}}=\frac{1}{n+1}+\sum_{j=1}^{j=n+1} \overline{p_{j i}}(n) \overline{e_{j}}
$$

Each node of the wireless network is similar, thus from symmetry $\overline{e_{i}}=\overline{e_{j}} \forall i ; j$. Also $\overline{p_{i j}}=\frac{1-p(n)}{n}\left(1-(1-A(n))^{n}\right)$. Since in our model $A(n)$ is chosen such that the network is connected with high probability, therefore $\left(1-(1-A(n))^{n}\right) \approx 1$ and hence $\overline{p_{i j}}(n) \approx \frac{1-p(n)}{n}$. From symmetry

$$
\overline{e_{i}}=\frac{1}{n+1}+\sum_{j=1, j \neq i}^{j=n+1} \frac{1-p(n)}{n} \overline{e_{i}}
$$


By rearranging the above equation we get (9).

Lemmas 1 and 2 (equations 8-9) indicate that the nodes visit ratio and the forwarding probabilities averaged over all possible instances of the topologies are similar to the visit ratios and forwarding probabilities of an average topology where each node has a number of neighbors equal to the average case. Thus, as a result of these two lemmas, one may derive the remaining set of model parameters (effective packet arrival rate and number of packets traversed) by considering the average case topology. Applying these results in the diffusion model will provide expressions for the average end to end delay, defined as the expectation of the packet delay over all packets and all possible networks.

Lemma 3 The effective packet arrival rate at a node $i$, denoted by $\lambda_{i}$, is

$$
\lambda_{i}=\lambda / p(n)
$$

Proof The packet arrival process at each node is an i.i.d. Poisson process with rate $\lambda$. So the total external arrival rate, denoted by $\lambda_{e}$, equals $(n+1) \lambda$. According to (2), $\lambda_{i}=\lambda_{e} e_{i}$. Substituting $\overline{e_{i}}$ from (9) and $\lambda_{e}$ we get (10).

Lemma 4 The expected number of hops traversed by a packet between its source and destination, denoted by $\bar{s}$, equals $\frac{1}{p(n)}$.

Proof Let $s$ denote the number of hops between a source and destination, then $P[s=k]=(1-p(n))^{k-1} p(n) k \geq 1$. Thus,

$$
\bar{s}=E[s]=\sum_{k=1}^{\infty} k \cdot(1-p(n))^{k-1} p(n)=\frac{1}{p(n)}
$$

The average queuing delay depends upon the service time distribution of the nodes. The service time distribution depends on the MAC protocol used by the nodes.

\section{QUEUING ANALYSIS}

In this section we first present a model for a random access MAC that accounts for the back off and collision avoidance mechanisms of IEEE 802.11 MAC. We then present the delay analysis of multihop wireless ad hoc networks by integrating the MAC model with the queuing network model developed in Section 3. 


\subsection{The MAC Model}

\subsubsection{Interfering Neighbors}

Two nodes are said to be interfering neighbors if they lie within a distance of $2 r(n)$ of each other. The transmission of a node would be successful if none of the interfering neighbors of the node transmits concurrently. Also two nodes may successfully transmit at the same time if they are not interfering neighbors of each other. The definition of interfering neighbors is similar to the definition given in [10].

\subsubsection{The Random Access MAC Model}

Before transmitting each packet the nodes count down a random back-off timer. The duration of the timer is exponentially distributed with mean $1 / \xi$. As in IEEE 802.11, the timer of a node freezes each time an interfering neighbor starts transmitting a packet. When the back off timer of a node expires, it starts transmitting the packet and the back off timers of all its interfering neighbors are immediately frozen. The timers of the interfering neighbors are resumed as soon as the transmission of the packet is complete. The time required to transmit a packet from a node to its neighbor is $L / W+T_{o}$, where $T_{o}$ is the time required for the exchange of RTS, CTS and ACK packets. We assume that $T_{o}$ is negligible compared to $L / W$, so in our analysis we assume that the time required to transmit a packet is $L / W$. The model is mathematically tractable and at the same time captures the behavior of IEEE 802.11 MAC protocol.

\subsection{Delay Analysis}

With the help of the following three lemmas we determine the mean and second moments of the service time of nodes using the random access MAC model. We then present the result for end-to-end delay in multihop wireless networks.

Lemma 5 Let $H_{i}$ denote the number of interfering neighbors of a node $i$. Then

$$
\begin{gathered}
E\left[H_{i}\right]=4 n A(n) \\
E\left[H_{i}^{2}\right]=4 n A(n)(1+4(n-1) A(n))
\end{gathered}
$$

where $A(n)=\pi \cdot r(n)^{2}$.

Proof Since the nodes are uniformly distributed over a unit area, the probability that a node is an interfering neighbor of node $i$ equals $\pi(2 r(n))^{2}$. Thus the proba- 
bility that $H_{i}=h$ is given by

$$
P\left[H_{i}=h\right]=\left(\begin{array}{l}
n \\
h
\end{array}\right)\left(4 \pi r(n)^{2}\right)^{h} \cdot\left(1-4 \pi r(n)^{2}\right)^{(n-h)}
$$

Thus $H_{i}$ has a binomial distribution. (12) and (13) are the first and second moment of the binomial distribution.

Lemma 6 Let $M_{i}$ denote the number of interfering neighbors of a node $i$ that have at least one packet to transmit. Then under steady state,

$$
\begin{gathered}
E\left[M_{i}\right]=\rho 4 n A(n) \\
E\left[M_{i}^{2}\right]=\rho^{2} \cdot 4 n A(n)(1+4(n-1) A(n))+(1-\rho) \rho 4 n A(n)
\end{gathered}
$$

where $\rho$ is the utilization factor of the nodes.

Proof Let the number of interfering neighbors of node $i$ be $H_{i}$. Let $Y_{j}, 1 \leq j \leq H_{i}$, be an indicator random variable associated with node $j$, indicating whether under steady state node $j$ has a packet to transmit or not. ( $Y_{j}=1$ if node $j$ has a packet to transmit, $Y_{j}=0$ if node $j$ has no packet to transmit). Using (5) $P\left(Y_{j}=1\right)=\rho_{j}$, where $\rho_{j}$ is the utilization factor of node $j$. By symmetry $\rho_{j}=\rho \forall j . M_{i}$ is equal to $\sum_{j=1}^{H_{i}} Y_{j}$. The expected value of $M_{i}$ equals

$$
E\left[M_{i}\right]=E_{H_{i}}\left[E\left[M_{i} \mid H_{i}=h\right]\right]=E_{H_{i}}\left[\sum_{j=1}^{h} E\left[Y_{j}\right]\right]=\rho E\left[H_{i}\right]
$$

Substituting (12), we get (14).

Similarly the expected value of $M_{i}^{2}$, given $H_{i}=h$, is given by

$$
E\left[M_{i}^{2} \mid H_{i}=h\right]=E\left[\left(\sum_{j=1}^{h} Y_{j}\right)\left(\sum_{k=1}^{h} Y_{k}\right)\right]
$$

Since $Y_{j}$ is independent of $Y_{k}$, we get

$$
\begin{array}{r}
E\left[M_{i}^{2} \mid H_{i}=h\right]=\sum_{j=1}^{h} \sum_{k=1, k \neq j}^{h} E\left[Y_{j}\right] E\left[Y_{k}\right]+\sum_{j=1}^{h} E\left[Y_{j}^{2}\right] \\
\Rightarrow E\left[M_{i}^{2}\right]=\rho^{2} E\left[H_{i}^{2}\right]+(1-\rho) \rho E\left[H_{i}\right]
\end{array}
$$

Substituting (12) and (13), we get (15).

Lemma 7 Let $Z_{i}$ denote the number of times the timer of a node $i$ is frozen before its expiration. Then

$$
E\left[Z_{i}\right]=4 \cdot \rho n A(n)
$$


Proof Let $T_{i}$ denote the duration of the back off timer of node $i$. During a transmission epoch $M_{i}$ may not remain constant. In order to simplify the analysis we assume that $M_{i}$ remains constant throughout a transmission epoch of node $i$. The timer of node $i$ is frozen each time a timer of any of the interfering neighbors of $i$ expires. The timer of each node has an exponential distribution. Thus the probability that $Z_{i}=z$, given that $M_{i}=m$ and $T_{i}=t_{i}$, is

$$
\begin{array}{r}
P\left[Z_{i}=z \mid T_{i}=t_{i}, M_{i}=m\right]=e^{-m \cdot \xi \cdot t_{i}} \cdot\left(m \cdot \xi \cdot t_{i}\right)^{z} / z ! \\
\Rightarrow E\left[Z_{i} \mid T_{i}=t_{i}, M_{i}=m\right]=m \xi t_{i} \\
\Rightarrow E\left[Z_{i} \mid M_{i}=m\right]=m \xi E\left[t_{i}\right]=m \Rightarrow E\left[Z_{i}\right]=E\left[M_{i}\right]
\end{array}
$$

Substituting $E\left[M_{i}\right]$ from (14), we get (16).

Theorem 1 Let $\overline{X_{i}}$ and $\overline{X_{i}^{2}}$ denote the mean and second moment of service time required to serve a packet by a node $i$. Then

$$
\begin{gathered}
\overline{X_{i}}=E\left[X_{i}\right]=\frac{\frac{1}{\xi}+\frac{L}{W}}{1-4 n A(n) \lambda_{i} \frac{L}{W}} \\
\overline{X_{i}^{2}}=E\left[X_{i}^{2}\right]\left(1+3 \bar{m}+2 \overline{m^{2}}\right) \frac{L^{2}}{W^{2}}+2(2 \bar{m}+1) \frac{L}{W} \frac{1}{\xi}+\frac{2}{\xi^{2}}
\end{gathered}
$$

where $\bar{m}=E\left[M_{i}\right]$ (eqn. (14)) and $\overline{m^{2}}=E\left[M_{i}^{2}\right]$ (eqn. (15)).

Proof The time taken by node $i$ to serve a packet, denoted by $X_{i}$, is the sum of three terms: (i) the duration of the random back off timer $\left(t_{i}\right)$, (ii) the duration for which the timer remains frozen $\left(Z_{i} L / W\right)$, and (iii) the transmission time $(L / W)$. Thus

$$
X_{i}=t_{i}+Z_{i} \frac{L}{W}+\frac{L}{W}
$$

Taking expectation of both sides we get,

$$
E\left[X_{i}\right]=E\left[t_{i}\right]+E\left[Z_{i}\right] \cdot \frac{L}{W}+\frac{L}{W}=\frac{1}{\xi}+4 \rho n A(n) \frac{L}{W}+\frac{L}{W}
$$

Substituting $\rho=\lambda_{i} \overline{X_{i}}$ and by rearranging, we get (18).

Also from (20) we have $X_{i}^{2}=\left(t_{i}+Z_{i}+\frac{L}{W}\right)^{2}$ Given $M_{i}=m$ and $T_{i}=t_{i}, Z_{i}$ has a Poisson distribution. So $E\left[Z_{i}^{2} \mid M_{i}=m, T_{i}=t_{i}\right]=m \xi t_{i}+\left(m \xi t_{i}\right)^{2}$. Using this and (17), we get

$$
E\left[X_{i}^{2} \mid T_{i}=t_{i}, M_{i}=m\right]=\left(1+\frac{L^{2}}{W^{2}} m^{2} \xi^{2}\right) t_{i}^{2}+\left(\frac{2 L}{W}+\frac{3 L^{2}}{W^{2}} m \xi\right) t_{i}+\frac{L^{2}}{W^{2}}
$$


Taking expectation with respect to $t_{i}$ we get

$$
\begin{array}{r}
E\left[X_{i}^{2} \mid M_{i}=m\right]=E_{T_{i}}\left[E\left[X_{i}^{2} \mid T_{i}=t_{i}, M_{i}=m\right]\right]= \\
\left(1+3 m+2 m^{2}\right) \frac{L^{2}}{W^{2}}+2(2 m+1) \frac{L}{W} \frac{1}{\xi}+\frac{2}{\xi^{2}}
\end{array}
$$

Taking expectation of the RHS w.r.t $m$, we get (19).

Corollary 1 The standard deviation of service time of a node $i$, denoted by $\sigma_{X_{i}}^{2}$, is given by

$$
\sigma_{X_{i}}^{2}=\frac{L^{2}}{W^{2}}\left(\bar{m}+\overline{m^{2}}+\sigma_{m}^{2}\right)+2(2 \bar{m}+1) \frac{L}{W} \frac{1}{\xi}+\frac{1}{\xi^{2}}
$$

where $\sigma_{m}^{2}=\overline{m^{2}}-\bar{m}^{2}$.

The squared coefficient of variance of the service time at a node $i$, denoted by $c_{B i}^{2}$ is given by $\sigma_{X_{i}}^{2} /{\overline{X_{i}}}^{2}$. Using (4), the squared coefficient of variance of the inter arrival time at a node $i$, denoted by $c_{A i}^{2}$, is given by

$$
c_{A i}^{2}=1+\sum_{j=1, j \neq i}^{n+1}\left(c_{B i}^{2}-1\right) \frac{1-p(n)}{n}=1+\left(c_{B i}^{2}-1\right)(1-p(n))
$$

With the knowledge of $c_{A i}^{2}, c_{B i}^{2}$ and $\rho$, we can find the parameter $\hat{\rho}$ as given in (6).

Theorem 2 For the random access MAC model the average end-to-end delay in a multihop wireless network, denoted by $D(n)$, is given by

$$
D(n)=\frac{\rho_{i}}{\lambda \cdot(1-\hat{\rho})}
$$

Proof Let $\overline{D_{i}}$ denote the average delay at a node $i$. According to Little's Law, $\overline{D_{i}}=\overline{K_{i}} / \lambda_{i}$, where $\overline{K_{i}}$ is the average number of packets in the queue of node $i$. Substituting $\overline{K_{i}}$ from (7) we get

$$
\overline{D_{i}}=\overline{K_{i}} / \lambda_{i}=\rho /\left(\lambda_{i}(1-\hat{\rho})\right)
$$

By symmetry the average delay at each node is same. Thus the average end-to-end delay equals the product of the average number of hops traversed by a packet and the average delay at each node. Hence $D(n)=\bar{s} \cdot \overline{D_{i}}$ which leads to (23).

\subsection{Maximum Achievable Throughput}

The maximum achievable throughput, denoted by $\lambda_{\text {max }}$, is defined as the maximum packet arrival rate at each node for which the average end-to-end delay remains 
finite. If the packet arrival rate exceeds $\lambda_{\max }$, the delay would tend to infinity. The following corollary, that follows from Theorem 1, yields a relationship between the maximum achievable throughput and the network parameters.

Corollary 2 For a multihop wireless network the maximum achievable throughput $\lambda_{\max }$ is

$$
\lambda_{\max }=\frac{p(n)}{\frac{1}{\xi}+\frac{L}{W}+4 n A(n) \frac{L}{W}}
$$

Also from (24), $\lambda_{\max }=o(1 / \bar{s} n A(n))$.

Proof From (18) the utilization factor of a node, $\rho_{i}$, is given by

$$
\rho_{i}=\lambda_{i} \cdot \frac{\frac{1}{\xi}+\frac{L}{W}}{1-4 n A(n) \lambda_{i} \frac{L}{W}}
$$

For the average delay to be finite $\rho_{i}$ must be strictly less than 1 . Thus the following inequality must be satisfied to ensure finite delay.

$$
\lambda_{i} \cdot \frac{\frac{1}{\xi}+\frac{L}{W}}{1-4 n A(n) \lambda_{i} \frac{L}{W}}<1
$$

Substituting $c=\frac{1}{\xi}+\frac{L}{W}, \lambda_{i}=\frac{\lambda}{p(n)}$ and rearranging, we get

$$
\lambda<\frac{p(n)}{c+4 n A(n) \frac{L}{W}}
$$

Thus the maximum achievable throughput $\lambda_{\max }$ is $\frac{p(n)}{c+4 n A(n) \frac{L}{W}}$. Also $c>0$, thus $\lambda_{\max }<\frac{p(n) W}{n A(n) L}$. So for a fixed packet size $L$ and transmission rate $W, \lambda_{\max }=$ $o\left(\frac{p(n)}{n A(n)}\right)$. Substituting $p(n)=\frac{1}{\bar{s}}, \lambda_{\max }=o\left(\frac{1}{\bar{s} n A(n)}\right)$.

The result of Corollary 2 re-emphasizes the importance of carefully choosing the transmission ranges of nodes. $\lambda_{\max }$ increases with decrease in $r(n)$. However if $r(n)$ is too small then the network would become disconnected. According to [13], the network is asymptotically connected for $r(n)=\omega(\sqrt{\log n / n})$. So for a connected network $A(n)=\omega(\log n / n)$ and $\lambda_{\max }=o\left(\frac{p(n)}{c+4 \log (n)(L / W)}\right)$.

Another interesting observation is the dependence of $\lambda_{\max }$ on the traffic pattern. $\lambda_{\max }$ is directly proportional to $p(n)$. From (11) the expected number of hops traversed by a packet equals $1 / p(n)$. Thus another way of interpreting the result is that $\lambda_{\max }$ is inversely proportional to the expected number of hops between a sourcedestination pair.

We further investigate how our result on the maximum achievable throughput compares with the result by Gupta-Kumar on throughput capacity. According to the 
Gupta-Kumar model, the nodes are distributed uniformly and independently over a sphere of unit surface area and each source chooses a random destination. Therefore the expected distance between a source and the corresponding destination equals the expected distance between two points uniformly and independently distributed on a sphere. Thus the expected distance between a source destination pair in GuptaKumar's model is a constant (i.e. does not vary with $n$ ), say $\overline{s_{G K}}$. The transmission range in their model is $\omega(\sqrt{\log n / n})$. Thus the expected number of hops between a source-destination pair in Gupta-Kumar model is $o(\sqrt{n / \log n})$.

In order to compare our results with Gupta-Kumar's results we choose our model parameters such that we have comparable average number of hops between a sourcedestination pair and comparable transmission range. In our model if we choose $p(n)=\sqrt{\log n / n}$, then the expected number of hops between a source and destination node is $\bar{s}=1 / p(n)=\sqrt{n / \log n}$, which is comparable to the Gupta-Kumar model. Also $r(n)=\sqrt{\log n / n}$ or $A(n)=\pi \log n / n$ makes the transmission range of our model comparable to that of the Gupta-Kumar model. So for the model parameters that are comparable to the Gupta-Kumar model, the maximum achievable throughput is

$$
\lambda_{\text {max }}=\frac{\frac{1}{4 \pi} \frac{W}{\sqrt{n \log n} L}}{1+\frac{c}{4 \pi \log n(L / W)}}
$$

or $\lambda_{\max }=o(W / \sqrt{n \log n})$.

The above discussion implies that for the similar values of parameters of the network model we get a bound similar to the Gupta-Kumar's bound on throughput capacity, but for our model the bound is not achievable. The reason for the bound not being achievable is that in our model we consider a random access MAC protocol rather than a perfect deterministic scheduling. Thus the bound becomes unachievable because some channel capacity is wasted by the nodes during contention for the channel.

\section{DISCUSSIONS}

In this section we present a brief intuitive interpretation of the mean service time and evaluate the maximum achievable throughput for multihop wireless networks. We also discuss how our analytical results vary from those obtained for a more pragmatic network model. 


\subsection{Interpretation of Mean Service Time}

We present a mathematically non-rigorous, but intuitive, derivation of mean service time of a node for the random access MAC model. This derivation further elucidates the result on service time. Consider a hypothetical two node network where one of the nodes transmits packets to the other node. Both nodes use the random access MAC model described in 4.1. In this scenario there is no contention for the channel and the average service time of the transmitter would be $\frac{1}{\xi}+\frac{L}{W}$. We refer to $\frac{1}{\xi}+\frac{L}{W}$ as the uncontended service time.

Now consider a node (say node 0 ) with $m$ interfering neighbors, numbered 1 through $m$. The node and its interfering neighbors use the random access MAC model for collision avoidance. Packets of size $L_{j}$ bits arrive at a rate of $\alpha_{j}$ packets/second at neighbor $j$. From the point of view of node 0 , the channel is available when no other interfering neighbor is transmitting. Under steady state, the fraction of time that the channel is available to node 0 is $1-\sum_{k=1}^{m} \alpha_{k}\left(L_{k} / W\right)$. So the service time of node 0 would be the uncontended service time scaled by the fraction of time the channel is available to node 0 . Hence the service time of node 0 equals $\frac{1 / \xi+L_{0} / W}{1-\sum_{k=1}^{m} \alpha_{k}\left(L_{k} / W\right)}$. We refer to $\sum_{k=1}^{m} \alpha_{k}\left(L_{k} / W\right)$ as the contention term.

In a multihop wireless network, $m$ is analogous to the number of interfering neighbors and $\alpha_{j}=\lambda_{i}, L_{j}=L \forall j$. The expected value of the contention term (or fraction of time channel is not available to a node) is $4 n A(n) \lambda_{i} \frac{L}{W}$ and therefore the service time of a node equals $\frac{1 / \xi+L / W}{1-4 n A(n) \lambda_{i}(L / W)}$.

\subsection{Comparison With Delay and Throughput in Real Networks}

The analytical model in this paper is kept reasonably simple so that it is possible to obtain closed form expressions for delay and throughput. In particular our MAC model does not take into account packet collisions and our routing model is random walk of packets over the network. Thus our model deviates from the real world scenarios where the packets collide due to random access MAC and the packets are routed along fixed paths dictated by routing protocols. In this subsection we discuss how much the delay and maximum achievable throughput in real world networks deviate from our analytical results.

\subsubsection{Effect of Packet Collisions}

Consider a more practical MAC model where a node transmits as soon as its transmit timer expires and the interfering neighbors freeze their timers only when they sense the transmission. For such a MAC, the transmission of node $i$ may collide 
with transmission of an interfering neighbor if difference between the time instances when the transmit timers of nodes $i$ and that of interfering neighbor expire is less than the propagation delay between the nodes. Let $d$ denote the propagation delay between node $i$ and its interfering neighbor that has a packet to send, then the probability that the transmission of $i$ does not collide with that of the interfering neighbor equals $e^{-2 \xi d}$. Since the interfering neighbors are located within two hops of node $i, d \leq 2 r / c=\delta$, where $v$ is velocity of electromagnetic waves. Thus the probability that the transmission of node $i$ does not collide with an interfering node's transmission is greater than $e^{-2 \xi \delta}$. So if node $i$ has $I$ interfering neighbors, then the probability that a transmission is a success is bounded by

$$
P[\text { Success }] \geq e^{-2 \xi \delta I}
$$

Let $P_{s}$ denote the expected probability of success, averaged over all possible topologies, then

$$
P_{s} \geq\left(1-\left(1-e^{-2 \xi \delta}\right) 4 A(n)\right)^{n}=P_{s}^{(L)}
$$

The expected number of times a node transmits a packet before it is received successfully by its neighbor equals $1 / P_{s}$. It is easy to see that the RHS of eqn. (21) is scaled by a factor of $1 / P_{s}$ and mean service time may be evaluated by rearranging the resulting equation. So for the more practical MAC model, that allows packet collisions, the mean service time is bounded by

$$
\frac{\frac{1}{\xi}+\frac{L}{W}}{1-4 n A(n) \lambda_{i} L / W} \leq \overline{X_{i}} \leq \frac{\frac{1}{\xi}+\frac{L}{W}}{P_{s}^{(L)}-4 n A(n) \lambda_{i} L / W}
$$

The maximum achievable throughput, evaluated using $\lambda_{i} \overline{X_{i}}<1$, is bounded by

$$
\lambda_{\max }^{(L)}=\frac{P_{s}^{(L)} p(n)}{\frac{1}{\xi}+\frac{L}{W}+4 n A(n) \frac{L}{W}} \leq \lambda_{\max } \leq \frac{p(n)}{\frac{1}{\xi}+\frac{L}{W}+4 n A(n) \frac{L}{W}}=\lambda_{\max }^{(U)}
$$

The dependence of $\lambda_{\text {max }}^{(L)}$, the lower bound of $\lambda_{\max }$, on the rate of transmit timer, $\xi$, is particularly interesting. As $\xi$ increases, both $P_{s}^{(L)}$ and $1 / \xi$ term in the denominator decrease. Thus there is a tradeoff in choosing the rate of the transmit timer - a high $\xi$ leads to lower waiting time before transmission but leads to higher probability of packet collision. Let $\xi^{\star}$ be the optimal value of $\xi$ that maximizes the lower bound of $\lambda_{\max }$. Equating $d \lambda_{\max }^{(L)} / d \xi$ to 0 yields that $\xi^{\star}$ satisfies the following relation

$$
\frac{\left(b(n) \xi^{\star 2}+\xi^{\star}\right) e^{-2 \xi^{\star} \delta}}{\left(1-4 A(n)\left(1-e^{-2 \xi^{\star} \delta}\right)\right)}=\frac{1}{8 n A(n) \delta}
$$

where $b(n)=L / W+4 n A(n) L / W$. Closed form expression for $\xi^{\star}$ cannot be evaluated from the above relation. However by approximating $e^{-2 \xi^{\star} \delta} \approx 1$ (high 
probability of success) and solving the resulting quadratic equation we get

$$
\xi^{\star} \approx \frac{1}{2 L / W} \frac{1}{1+4 n A(n)}\left(\sqrt{1+\frac{(1+4 n A(n)) L}{2 n A(n) W \delta}}-1\right)
$$

As expected, $\xi^{\star}$ decreases with increase in the expected number of interfering neighbors, packet transmission time and propagation delay.

\subsubsection{Effect of Deterministic Routing}

In the routing model used in this paper, a node forwards a packet to any of its neighbor with equal probability which spreads the traffic evenly throughout the network. On the other hand, a deterministic routing protocol routes each packet belonging to a particular flow (typically identified by source-destination pair) along a deterministic path, determined using some goodness metric. This often leads to a situation where large number of flows pass through a few nodes that have good paths to many flow destinations. This leads to creation of routing bottlenecks leading to large queuing delays intermediate nodes and higher end-to-end delays. Thus (23) is a lower bound on the average end-to-end delays in networks with deterministic routing.

\section{SimulationS}

We perform the following simulations:

(1) Simulations of the model: These simulations verify the validity of the assumptions made in the analysis and the accuracy of diffusion analysis.

(2) Simulations using shortest path routing instead of probabilistic routing: We compare the analytical results with a practical scenario where the packets are routed along the shortest path rather than undergoing probabilistic routing.

(3) NS simulations: These simulations provide comparison of the results of our analytical model against results obtained from NS simulations that employ standard MAC (IEEE 802.11) and routing (DSDV) protocols.

The rest of this section presents the simulation results for each of the above scenarios. 


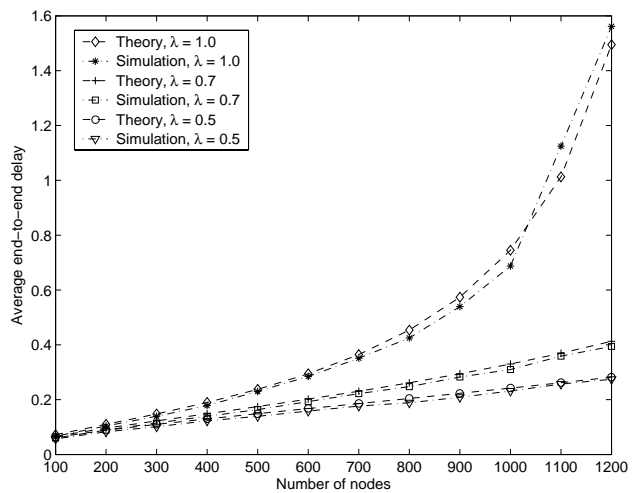

(a) Average end-to-end delay vs. number of nodes for varying arrival rates.

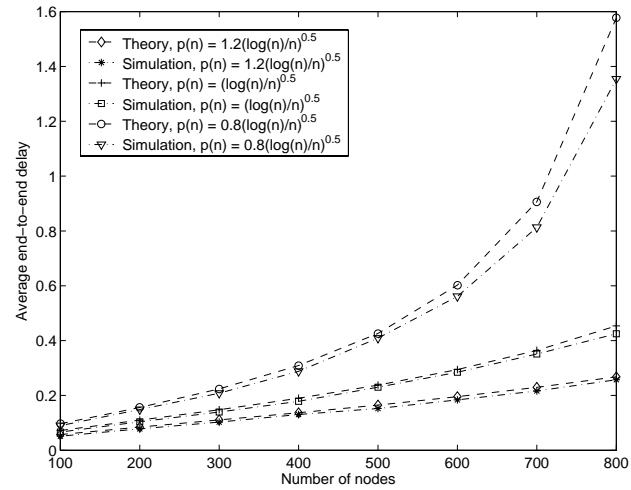

(c) Average end-to-end delay vs. number of nodes for varying absorption probabilities.

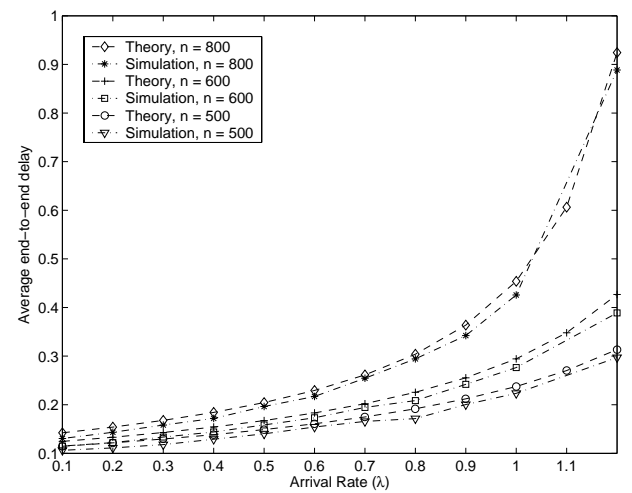

(b) Average end-to-end delay vs. arrival rate for varying network size.

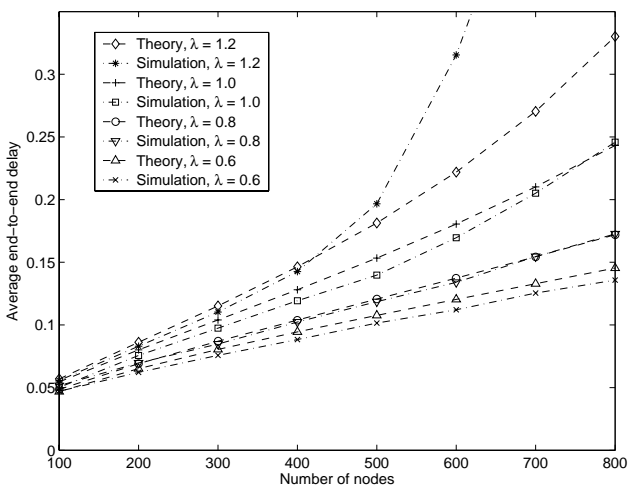

(d) Comparison of analytical results with simulation results for the shortest path routing.

Fig. 3. Comparison of the analytical results with simulation results.

\subsection{Simulations for Validating the Analytical Results}

The simulation setting is the following. The network topology for the simulations consists of $n$ nodes scattered randomly over a torus of unit surface area. Each node can communicate with the nodes within a distance $r(n)=\sqrt{\frac{\log n}{n}}$. The random access MAC protocol used by the nodes is the same as described in 4.2. Each node produces packets of size $L=1 \mathrm{Kbits}$ at the rate of $\lambda$ packets/sec. The transmission rate of each node is $W=10^{6} \mathrm{bits} / \mathrm{sec}$. The probabilistic routing described in Section 3 is used for the simulations. In order to ensure that the network is in a steady state, the first 100 seconds of the simulations are discarded. The average delay for a particular topology is obtained by averaging the end-to-end delay of all packets produced during the simulation. In order to average out the effect of topology, each simulation is repeated over several topologies. The average end-to-end delay 
is obtained by averaging the average delay for all topologies.

Figure 3(a) shows how the average end to end delay, as obtained from the simulations, varies with the number of nodes for $\lambda=0.5, \lambda=0.7$ and $\lambda=1.0$ with $p(n)=\sqrt{\frac{\log n}{n}}$. Figure 3(b) shows how the average end to end delay varies with the arrival rate $(\lambda)$ for $n=500,600$ and 800 with $p(n)=\sqrt{\frac{\log n}{n}}$. Figure 3(c) shows how the average end to end delay varies with the number of nodes for various values of absorption probability with $\lambda=1$ packets/sec. The theoretical values of the average end-to-end delay as obtained from the analytical results are plotted alongside the simulation results in Figures 3(a), 3(b) and 3(c). It is observed that the simulation results agree closely with the theoretical values.

\subsection{Comparison of Results for the Shortest Path Routing with the Analytical Re- sults}

In our model the packets are subjected to probabilistic routing, which is similar to a random walk. It would be pertinent to compare the analytical results for our model with the simulations where the packets are routed to the destination along the shortest path.

The simulation setting differs from the setting described in the previous subsection in the following manner. When a new packet is generated at a node, a destination node for the packet is chosen at random. The packet is routed to the destination node along the shortest path. In order to route the packets along shortest paths, each node maintains a routing table. The routing tables are constructed using Bellman Ford algorithm.

Figure 3(d) shows the simulation results for the shortest path routing along with the analytical results obtained from our model. The absorption probability of our model is scaled appropriately so that the average number of hops between a sourcedestination pair for the shortest path routing matches with the average number of hops traversed by a packet in the probabilistic routing model. It is observed that when the network is lightly loaded, the analytical results closely approximate the average delay with the shortest path routing. However as the network becomes loaded the average delay obtained from the simulations is much higher than the analytical results. The reason for this deviation is that in the case of the shortest path routing bottlenecks are formed in the network in highly loaded conditions. The probabilistic routing ensures that the traffic is spread uniformly over the network and thus the average end-to-end for the analytical model increases less rapidly. 


\subsection{Comparison Against Results from NS Simulations}

As mentioned earlier, the aim of the delay analysis presented in this paper is to capture the effect of random access MAC and queuing delays on the average end to end delay and maximum achievable throughput of multihop wireless networks. Our model includes an idealistic random access MAC and probabilistic routing model. The MAC model assumes that the transmission timers of all interfering neighbors of a node freeze as soon as the node starts transmitting. This precludes the possibility of any packet collision at the intended receiver. However IEEE 802.11, which is the de facto MAC protocol of wireless networks, is not free from collisions. The RTS packets transmitted by a node may collide with a transmission at the intended receiver which would then prevent the node from grabbing the channel. (This is more likely in networks with large contention window, e.g. long propagation delays.) Also when a node, in an ad-hoc IEEE 802.11 network, starts transmitting, the transmission timers of only a subset of interfering neighbors are frozen. This subset includes only the neighbors of the transmitter and the intended receiver.

In this section we compare the analytical results for our model with the average delay obtained from NS-2 simulations, that use IEEE 802.11 as MAC and DSDV for routing. The purpose of this comparison is to understand how the end to end delay in a network based on the established protocols would differ from the results obtained from our model, given that our model includes some simplifying assumptions that does not capture the actual protocols (and their interactions).

The NS simulation set-up is as follows. The network consists of $n$ nodes that are uniformly and independently distributed over a $500 \mathrm{~m} \times 500 \mathrm{~m}$ area. An exponential traffic source is attached to each node, which produces packets of length 1000 bytes at the rate of $\lambda$ packets per second. Each node chooses a random destination and the traffic is routed to the destination using routes maintained by DSDV $^{3}$. UDP is used as the transport layer protocol in order to avoid delays due to congestion avoidance mechanisms of TCP. The receive threshold ${ }^{4}$ of the nodes is set such that each node within a distance of $500 \cdot \sqrt{\log n / n}$ meters from a transmitter is able to listen to the transmission, in absence of any interference. The IEEE 802.11 MAC and free space propagation models are used for the simulations.

In order to compare the simulation results with the analytical results we set the values of the parameters of the analytical model such that they are comparable to that of the simulation. This is done in the following manner. We obtain the values of the average duration of the IEEE 802.11 backoff timer $\left(\xi^{\prime}\right)$ and the transmission duration $\left(T_{o}^{\prime}\right)^{5}$ for each simulation setting. We use $\xi=\xi^{\prime}$ and $\frac{L}{W}=T_{o}^{\prime}$ in the

\footnotetext{
3 Since the nodes are stationary, DSDV is the ideal routing protocol.

4 If the signal strength at a receiver is below receive threshold, the receiver cannot detect the signal. Please see [7] for details.

${ }^{5} T_{o}^{\prime}$ includes the time taken for the exchange of RTS, CTS, data and ACK packets i.e.
} 


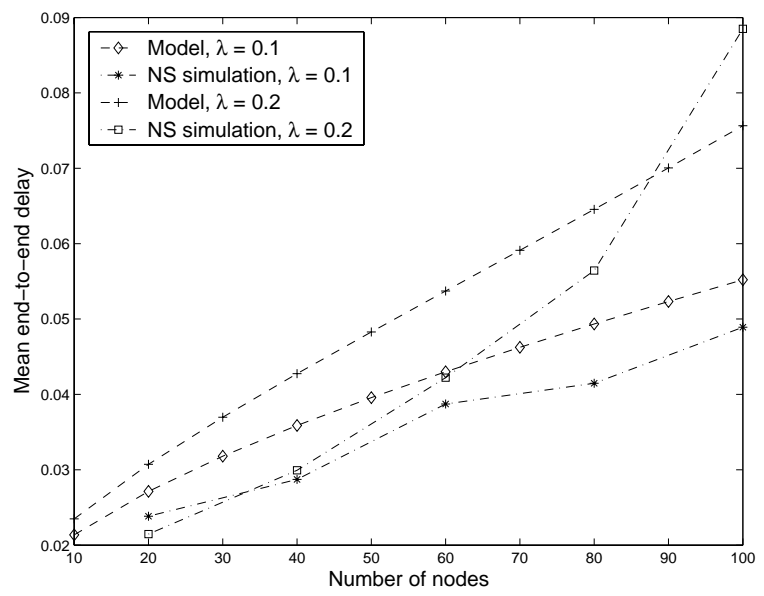

Fig. 4. Average end-to-end delay vs. number of nodes for NS simulations.

analytical results. This ensures that the average backoff duration of MAC protocol and the transmission time of the analytical model is same as that of the simulations.

Figure 4 shows the plots of average end-to-end delay, obtained from NS-2 simulations and analytical model, as a function of number of nodes. We observe that for lightly loaded conditions ( small $\lambda$ and/or $n$ ) the simulation results are less than the analytical results. This is because in IEEE 802.11 only the transmission timers of the neighbors of the transmitter and receiver are frozen during a transmission while in our model we assume that the timers of all interfering neighbors are frozen. Thus the number of times the transmission timer of a node is frozen in IEEE 802.11 is smaller than that in our model. So the average time in which the transmission timer of a node expires is less in IEEE 802.11 leading to lesser delays. However as the traffic load in the network increases the average end-to-end delay of the simulation becomes larger than the analytical results. This is because the number collisions in the simulations increase sharply as the network load increases. The number of collisions is further increased due to the broadcast of routing update packet which are not preceded by virtual carrier sense and RTS-CTS exchange [6]. The delay is also affected by the formation of routing hot spots in heavy load conditions. The simulation results indicate that the capacity of wireless networks based on standard protocols is significantly lower than that predicted by our model. The decreased in capacity is caused due to the collision of packets and broadcast of routing updates.

\section{CONCLUSION AND FUTURE WORK}

Characterization of capacity and delay in ad hoc networks has been focus of considerable research. However capacity and delay of networks based on random access MAC, like IEEE 802.11, have not been substantially studied. In this paper

$\overline{T_{o}^{\prime}=T_{\mathrm{RTS}}}+T_{\mathrm{SIFS}}+T_{\mathrm{CTS}}+T_{\mathrm{SIFS}}+T_{\mathrm{DATA}}+T_{\mathrm{SIFS}}+T_{\mathrm{ACK}}$. 
we presented delay analysis of random access MAC multihop wireless ad hoc networks. We derived closed form expressions for the average end-to-end delay and maximum achievable throughput. We showed that, for comparable network parameters, the upper bound on maximum achievable throughput is of the same order as the Gupta-Kumar's bound. However for the random access MAC the bound is not achievable. The analytical results are verified using simulations. The NS-2 simulations indicate that under heavy load the performance of the standard wireless protocols is worse than the performance predicted by our model.

The results and framework presented in this paper leads to several venues for future research. Our current directions include the delay analysis and characterization of the maximum achievable throughput for hierarchical networks, many to one communication scenarios, wireless networks with sleeping nodes and wireless networks with other medium access control algorithms.

\section{ACKNOWLEDGEMENT}

This work was funded in part by the National Science Foundation under grants CNS-322956 and CNS-546402.

\section{References}

[1] D. Bertsekas and R. Gallager. Data Networks. Prentice-Hall, 1992.

[2] V. Bharghavan, A. Demers, S. Shenker, and L. Zhang. MACAW: A media access protocol for wireless LANs. In Proceedings of the Conference on Communications Architectures, Protocols and Applications, pages 212-225. ACM Press, 1994.

[3] G. Bianchi. Performance analysis of the IEEE 802.11 distributed coordination function. IEEE Journal on Selected Area in Communications, 18:535-547, March 2000.

[4] G. Bolch, S. Greiner, H. de Meer, and K. S. Trivedi. Queuing Networks and Markov Chains, chapter 10, pages 423-430. John Wiley and Sons, 1998.

[5] M. M. Carvalho and J. J. Garcia-Luna-Aceves. Delay analysis of IEEE 802.11 in single-hop networks. In ICNP '03: Proceedings of the 11th IEEE International Conference on Network Protocols, page 146, Washington, DC, USA, 2003. IEEE Computer Society.

[6] J.-M. Choi, J. So, and Y.-B. Ko. Numerical analysis of IEEE 802.11 broadcast scheme in multihop wireless ad hoc networks. In ICOIN'05: Proceedings of the International Conference on Information Networking 2005, pages 1-10, 2005. 
[7] K. Fall and K. Varadhan. The NS-2 user manual. http://www.isi.edu/nsnam/ns/.

[8] A. E. Gamal, J. Mammen, B. Prabhakar, and D. Shah. Throughput-delay trade-off in wireless networks. In Proceedings of IEEE INFOCOM (INFOCOM'04). IEEE, March 2004 .

[9] A. L. Garcia. Probability and Random Processes for Electrical Engineering, chapter 9, pages 499-558. Addison Wesley Longman, 1994.

[10] P. Gupta and P. R. Kumar. Capacity of wireless networks. IEEE Trans. on Information Theory, pages 388-404, March 2000.

[11] P. Karn. MACA: a new channel access methos for packet radio. In Proceedings of the 9th Comouter Networking Conference, pages 134-140, September 1990.

[12] P. Kouvekis and D. Tripathi. Approximate performance modeling and decision making for manufacturing systems, a queuing network optimization framework. Journal of Intelligent Manufacturing, 2:107-134, 1991.

[13] A. Kumar, D. Manjunath, and J. Kuri. Communication Networking An Analytical Approach, chapter 8, pages 456-476. Morgan Kaufman Publishers, 2004.

[14] J. Li, C. Blake, D. S. D. Couto, H. I. Lee, and R. Morris. Capacity of ad hoc wireless networks. In MobiCom '01: Proceedings of the 7th annual international conference on Mobile computing and networking, pages 61-69, New York, NY, USA, 2001. ACM Press.

[15] X. Lin and N. B. Sheroff. Advances in Pervasive Computing and Networking, chapter 2, pages 17-55. Springer Science, New York, NY, 2004.

[16] S. McCanne and S. Floyd. NS Network Simulator. http://www.isi.edu/nsnam/ns/.

[17] D. Miorandi, A. A. Kherani, and E. Altman. A queueing model for HTTP traffic over IEEE 802.11 WLANs. In Proceedings of 16th ITC Specialist Seminar on Performance Evaluation of Wireless and Mobile Systems, August 2004.

[18] M. Ozdemir and A. B. McDonald. An M/MMGI/1/K queuing model for IEEE 802.11 ad hoc networks. In Proceedings of the 1st ACM International Workshop on Performance Evaluation of Wireless Ad Hoc, Sensor, and Ubiquitous Networks, pages 107-111. ACM Press, 2004.

[19] S. Ray, D. Starobinski, and J. B. Carruthers. Performance of wireless networks with hidden nodes: A queuing-theoretic analysis. To appear in Journal of Computer Communications.

[20] M. Reiser and H. Kobayashi. Accuracy of the diffusion approximation for some queueing systems. IBM Journal of Research and Development, 18:110-124, 1974.

[21] S. D. Servetto and G. Barrenechea. Constrained random walks on random graphs: routing algorithms for large scale wireless sensor networks. In WSNA '02: Proceedings of the 1st ACM international workshop on Wireless sensor networks and applications, pages 12-21, New York, NY, USA, 2002. ACM Press. 
[22] O. Tickoo and B. Sikdar. On the impact of IEEE 802.11 MAC on traffic characteristics. IEEE Journal on Selected Areas in Communications, 21(2):189-203, 2003.

[23] O. Tickoo and B. Sikdar. A queueing model for finite load IEEE 802.11 random access MAC. In To appear in the proceedings of IEEE ICC, Paris, France, June 2004.

[24] G. Zeng, H. Zhu, and I. Chlamtac. A novel queueing model for 802.11 wireless LANs. In Proceedings of WNCG Wireless Networking Symposium, 2003.

Nabhendra Bisnik received the B.Tech. in electrical engineering from Indian Institute of Technology, Kanpur in 2003. He is currently working towards the Ph.D. Degree at the Rensselaer Polytechnic Institute, Troy, NY. His research interests include modeling and analysis of wireless ad hoc networks, phenomena coverage problems in wireless sensor networks and resource discovery in dynamic networks.

Alhussein A. Abouzeid received the B.S. degree with honors from Cairo University, Cairo, Egypt in 1993, and the M.S. and Ph.D. degrees from University of Washington, Seattle, WA in June 1999 and July 2001, respectively, all in electrical engineering. From 1994 until 1997, he served as a Project Manager in the Middle East Regional Office of Alcatel telecom. From 1998 to 2001, he was a Research Assistant in the Department of Electrical Engineering, University of Washington, Seattle, WA. His research collaborations during this period included Honeywell (previously AlliedSignal), Redmond, WA, HRL, Malibu, CA and Microsoft Research, Redmond, WA. Starting from August 2001, he assumed his current position as Assistant Professor in the Electrical, Computer and Systems Engineering Department at Rensselaer Polytechnic Institute, Troy, NY. His research interests include stochastic modeling of transport protocols, modeling active queue management in the Internet, and information extraction, routing, and self-configuration in large-scale variable topology networks, including wireless sensor networks and overlay (P2P) networks. His teaching contributions include the development of a graduate-level wireless networks course at RPI, with a particular focus on wireless ad-hoc networks. He received the Faculty Early Career Development Award (CAREER) from NSF in 2006. He is a member of IEEE and ACM. 